\title{
The Syntax of simple and compound tenses in Ndebele
}

\author{
Joanna Pietraszko*
}

\begin{abstract}
It is a widely accepted generalization that verbal periphrasis is triggered by increased inflectional meaning and a paucity of verbal elements to support its realization. This work examines the limitations on synthetic verbal forms in Ndebele and argues that periphrasis in this language arises via a last-resort grammatical mechanism. The proposed trigger of auxiliary insertion is c-selection - a relation between inflectional categories and verbs.
\end{abstract}

Keywords. verbal periphrasis; default auxiliary; c-selection; Ndebele; Bantu

1. Introduction. This paper focuses on the mechanics of default periphrasis - a phenomenon where a default verb (an auxiliary) appears in a complex inflectional context. I provide an analysis of two types of compound tenses in Ndebele: ${ }^{1}$ Perfect and Prospective tenses, arguing that periphrastic expressions in those tenses are triggered by c-selectional features on $\mathrm{T}$ heads. The type of verbal periphrasis we find in Ndebele is known as the overflow pattern of auxiliary use (Bjorkman, 2011), and is illustrated in (1), ${ }^{2}$ where Present Perfect and Simple Future are synthetic (there is no auxiliary), but Future Perfect is periphrastic (1-c).

Default periphrasis in Ndebele:
a. U- $\varnothing-$ dl -ile
Present Perfect/Recent Past (synthetic)
2sg- PST- eat -FS.PST
'You have eaten/you ate recently'.
b. U- Za- dl -a
2sg- FUT- eat -FS
'You will eat'.
$\begin{array}{lll}\text { c. } & \text { U- } \text { za- be } & \text { u- } \varnothing-\text { dl -ile } \\ \text { 2sg- FUT- aux } & \text { 2sg- PST- eat -FS.PST }\end{array}$
'You will have eaten'.
Simple Future (synthetic)
Future Perfect (periphrastic)

The characteristic feature of the overflow pattern is that the verb does not discriminate what inflection it can or cannot combine with. As we see in (1-a) and (1-b), the verb can combine with both perfect ${ }^{3}$ and future inflections to form a synthetic expression. What triggers periphrasis, is their co-occurrence - the verb cannot host both at the same time, as we see from the periphrastic form in (1-c). This generalization is not true of the additive pattern of auxiliary use (Bjorkman, 2011), found e.g. in English, where the Perfect always requires auxiliary syntax. One goal of this paper is to provide an account of the understudied overflow pattern.

It is well known that the distribution of periphrastic forms is not arbitrary crosslinguistically: present tenses are typically synthetic, but the Future Perfect, for instance, tends to require

\footnotetext{
* I'd like to thank my Ndebele consultants: Lily Dubé , Galen Sibanda and Nkululeko Sibanda. For helpful comments and discussions I'm grateful to Karlos Arregi, David Adger, Ben Bruening, Itamar Francez, John Goldsmith, Jason Merchant, David Pesetsky and the audience of LSA 90. Author's affiliation: University of Chicago (pietraszko@uchicago.edu)

${ }_{1}$ Bantu, Zimbabwe (Guthrie classification: S44).

2 Abbreviations: $2 \mathrm{sg}=$ 2nd person singular subject agreement, $\mathrm{FS}=$ final suffix, FUT $=$ future tense, $\mathrm{PST}=$ past tense.

${ }^{3}$ Present Perfect is morphologically identical to the Recent Past - a connection that will become relevant in sec. 4.
} 
an auxiliary. The various accounts of those tendencies offered in the literature follow the strong intuition that periphrasis occurs with an increased amount of inflectional features (e.g. when both future tense and perfect aspect are to be expressed) and a paucity of inflection hosts, i.e. verbs. This intuition raises a fundamental question about periphrasis: Why is it that "inflectional overflow" is repaired by the presence of an additional verb? Most work on periphrasis sets off with the assumption that inflectional features must combine with a verb. The term "combine" has been understood it different ways (morphological, syntactic), but the general idea is simple: inflection needs a verb. The logic of this generalization is schematized (2) and (3).
a. * Infl
b. $\quad \checkmark$ Infl $\mathrm{V}$

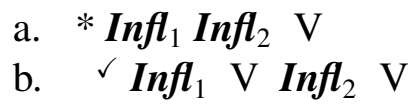

(2) reflects the assumption that inflection must combine with a verb. (3) represents periphrasis, for instance the Future Perfect in Ndebele. I argue that understanding why auxiliaries appear is to understand the formal relationship between inflection and verbs in general. In other words, we should understand (3) as an instance of (2). This is the second goal of this paper.

The proposed connection between auxiliary verbs and main verbs is simply that they are verbs, and as such, are both c-selected by inflectional categories. That is, both (2) and (3) are accounted for by the c-selectional requirement of an inflectional head. Thus, the driving force behind auxiliary insertion (3) is the general relationship between verbs and inflection (2).

I begin in the next section by discussing the rationale behind the last-resort view of auxiliary use and briefly reviewing previous accounts of periphrasis in these terms. In section 3, I detail the mechanics of periphrasis in general terms, arguing that auxiliary insertion can be understood in terms of independently motivated syntactic operations: c-selection formalized as Agree (Svenonius, 1994; Cowper, 2010) and the Cyclic Agree view of agreement (Béjar \& Rezac, 2009). In section 4, I apply this mechanism to the overflow pattern of periphrasis in Ndebele, deriving the overflow in tense features (Perfect and Prospective tenses). Section 5 evaluates the proposed analysis, as well as two existent alternative accounts, against the two objectives discussed above. Section 6 concludes the paper.

2. Default periphrasis as a last-resort phenomenon. Recent work on inflectional periphrasis cross-linguistically has produced a number of analyses of periphrasis as a last-resort mechanism (Déchaine, 1995; Schütze, 2003; Cowper, 2010; Bjorkman, 2011; Arregi \& Klecha, 2015). The basic logic underlying this approach is that auxiliaries do not appear unless necessary. More specifically, an auxiliary verb is used always and only when an inflectional feature cannot combine with the main verb. As an illustration, consider the contrast between (4) and (5).
Auxiliary 'be' not required
a. John work-s. $\checkmark$
b. *John is work.

Auxiliary 'be' required

a. *John working-s.

b. John is working. $\checkmark$

From the grammatical (4-a) we see that the suffix $-s$ can combine with the verb 'work' to form a synthetic expression. The last-resort profile of periphrasis in (4) is evident from the ungrammaticality of (4-b): where unnecessary, the auxiliary is not allowed. The progressive participle, on the other hand, resists combining with the present tense suffix (5-a). As a last resort way of expressing that feature, the auxiliary be must be inserted and support its realization. However, the last-resort effect we observe in (4) and (5) does not require an analysis based on a last-resort grammatical mechanism. In fact, most standard analyses of compound tenses in English, like the 
progressive tenses, do not implement last resort in the theoretical sense. I briefly discuss these standard accounts of English in the next subsection and in subsection 2.2. I show why they are incompatible with the overflow pattern.

2.2. The ADDitive PATTERn. There is convincing evidence that, unlike lexical verbs, default auxiliaries, such as English be, do not contribute to the semantics of the predicate (Chomsky (1993); Rothstein (1999, 2004); Iatridou et al. (2003); Pancheva (2003), among others). For instance, auxiliary verbs do not seem to have their own thematic structure. Rather, all arguments that occur in a clause with an auxiliary verb are arguments of the main, lexical verb. Given the apparent semantic vacuity of auxiliary verbs, it is a common claim that their occurrence is triggered by formal constraints of the grammar. Partly for this reason, default auxiliaries have been analyzed as i) semantically empty Vs projecting their own VPs within the functional domain (Dechaine1995, Iatridou et al. 2003; Pancheva 2003, among others) or realizations of certain functional heads, rather than as separate verbal projections (Cinque, 1999). These two types of analysis of the English auxiliary be are in (6) an (7), illustrated with progressive aspect.

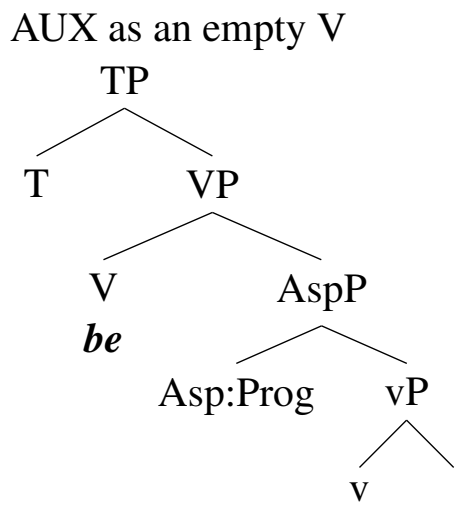

AUX as a functional head

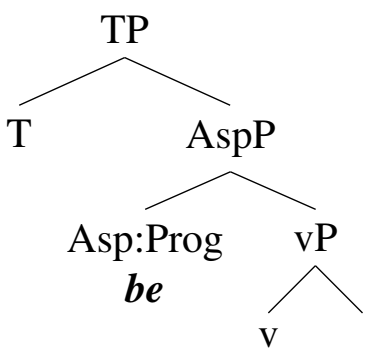

According to the "empty-V" approach in (6), the auxiliary verb is merged in the course of syntactic derivation and projects its own VP. Under this view, the question arises of what determines whether an empty $\mathrm{V}$ will be merged at some level of the inflectional structure. It is typically assumed that the $\mathrm{V}_{\text {Aux }}$ selects a particular inflectional feature, for instance the progressive Asp in English. The selection-based connection between the auxiliary and some inflectional categories accounts for their distributional connection: the two always co-occur in English. Another way to account for the systematic co-occurrence of the auxiliary be with progressive aspect is to posit that the auxiliary is a realization of the progressive head - this is the functional-head analysis in (7). Under this view, auxiliaries do not project their own phrases, but simply spell out functional heads that correspond to the relevant inflectional categories, such as Asp with the value Prog.

Importantly, neither of these accounts involves any kind of last-resort mechanism deriving auxiliary instertion. The auxiliary is necessarily present in both (6) and (7) - there are no conditions under which either of the structures could give rise to synthetic forms. This type of analysis is compatible with the additive pattern of auxiliary use, found in English, where a particular inflection (here, the progressive aspect) never appears without an auxiliary. As we shall see in the next section, the overflow pattern is inherently incompatible with either (6) or (7), and requires a last-resort type of derivation.

2.3. THE OVERFLOW PATTERN. In some languages, auxiliaries do not systematically co-occur with a particular inflection. Instead, periphrasis triggered by a combination of inflectional heads. 
Consider again the overflow periphrasis in Ndebele:

\section{The overflow pattern in Ndebele}

a. U- $\varnothing-$ dl -ile

2sg- PST- eat -FS.PST

'You have eaten'.

b. U- $\mathrm{Za}-\mathrm{dl}-\mathrm{a}$

2sg- FUT- eat -FS

'You will eat'.

$\begin{array}{llll}\text { c. } & \text { U- } \\ \text { 2a- be } & \text { u- } & \varnothing-\text { dl -ile } \\ \text { 2sg- FUT- aux } & \text { 2sg- PST- eat -FS.PST }\end{array}$

'You will have eaten'.

Simple Future (synthetic)

Present Perfect (synthetic)

Future Perfect (periphrastic)

(9) $\quad[\mathrm{T} \quad[$ Perf $[\mathrm{v}$ verb $]]]$

Auxiliary distributions like the one in (8) were extensively discussed by Bjorkman (2011) as an argument against the standard analyses of verbal periphrasis in (6) and (7). The incompatibility has to do with a type of look-ahead problem. Since the perfect inflection does not, in principle, require an auxiliary (8-a), at the point in the derivation where the perfect aspect is merged, it cannot be known if the structure will require an auxiliary or not. This can only be determined when $\mathrm{T}$ is merged, and if it is future (or non-present, in general), the auxiliary will surface (8-c). Unlike (6) and (7), then, the structure in (9) is not unambiguously synthetic or periphrastic. Thus, the overflow pattern forces an analysis that implements a last-resort mechanism of auxiliary insertion that applies in the course of the derivation.

Recent literature offers a series of accounts of inflectional periphrasis as a last-resort mechanism (Schütze, 2003; Cowper, 2010; Bjorkman, 2011; Arregi \& Klecha, 2015): accounts where $\mathrm{V}_{\text {Aux }}$ neither projects a VP, nor spells out a functional head. All these accounts rely on the assumption that inflection must combine with a verb. They differ in how they treat this relationship: as a syntactic one (Cowper, 2010; Arregi \& Klecha, 2015) or as a morphological one (Schütze, 2003; Bjorkman, 2011). This choice depends in part on our understanding of the term inflection, as either a morphosyntactic feature/functional head or an affix, a morpho-phonological object. If we understand inflection as an abstract syntactic object, then the nature of the verb-infl relationship must be one that can combine syntactic objects, for instance the syntactic operations Agree or Merge. Under the latter understanding of inflection, as an affix, the process of combining inflections with verbs would possibly be a morphophonological process. I will follow the work on periphrasis cited above in that the way inflections combine with verbs is a syntactic process targeting morphosyntactic features, rather than phonological exponents of particular inflectional morphemes.

The basic idea behind the last-resort accounts is that periphrasis is treated as a repair strategy whereby a default verb (the auxiliary) is inserted in case some inflectional category cannot combine with the main verb. Setting aside the analytical details, the general claim is that an intervening functional head, e.g. Perf in (8-c), disrupts the relationship between a higher functional head $\left(\mathrm{T}_{\mathrm{Fut}}\right.$ in $\left.(8-\mathrm{c})\right)$ and the main verb, preventing the tense inflection from combining with the verb (10). 

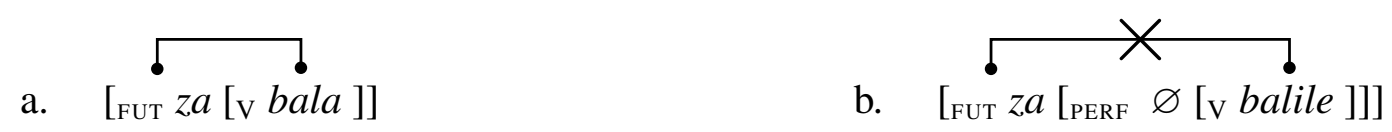

It is worth noting that the violation in (10-b) cannot be seen as purely morphophonological - the lower past tense has a null exponent and nonetheless prevents concatenation of the future tense prefix $z a$ - on the verb stem. Auxiliary insertion is then triggered by co-occurrences of morphosyntactic features, rather than overt affixes. If a functional head is not sufficiently local to an element of verbal category, the stranded inflection must be supported by a default verb - the auxiliary.

Ndebele periphrastic tenses, which exhibit the overflow pattern, must be derived by a lastresort mechanism of the general type in (10). In the next section, I propose that this mechanism is c-selection and present the details of its application.

3. Proposal: Auxiliary insertion as selection-triggered Merge. The proposed relationship between inflections and (auxiliary) verbs is that of c-selection: functional heads in the extended projection of the verb c-select for the verbal category. This is the relationship that is responsible for auxiliary insertion in contexts where some inflectional feature cannot combine with the main verb. While the link between auxiliary insertion and c-selection has been previously proposed for English (Cowper, 2010; Déchaine, 1995), the mechanism of auxiliary insertion developed there is incompatible with the type of periphrasis found in Ndebele, namely the overflow pattern. I argue that auxiliary verbs, just like main verbs, are c-selected by inflectional heads. Unlike main-verb selection, however, auxiliary insertion is a last-resort mode of satisfying selectional features on functional heads.

3.1 STRUCTURE BUILDing: C-SELECTION AND FUnCTIONAL HIERARCHIES. Following Cowper (2010), I formalize c-selection as an agreement relation (established by the operation Agree). In Cowper's terms, the probing heads are inflectional heads with uninterpretable V-features, which must be checked against a matching interpretable goal - a verb. I propose a different formalization, though this particular implementation is not essential for the general account proposed here.

I propose that c-selectional features have the form of category probes - unvalued category features [Cat:_]. The value is provided by a category goal, an element with a valued category feature. Verbs have the category feature valued for V ([Cat: V]), while nouns, for instance, have [Cat: N]. In principle, then, any element with a valued category feature could control agreement on the probe. Since c-selection, by definition, discriminates between categories, any Agree-based account of selection must impose some sort of matching requirement. I implement this matching requirement as relativized probing: each category probe is relativized for a particular category (indicated by a subscript on the probe). As shown in (11), the selecting head Y has an unvalued category probe relativized for X, which means it c-selects for X. Upon Merge with an element of category $\mathrm{X}$, the unvalued category feature on $\mathrm{Y}$ probes, finds $\mathrm{X}$ and copies its [Cat]-value.

C-selection as category probing:
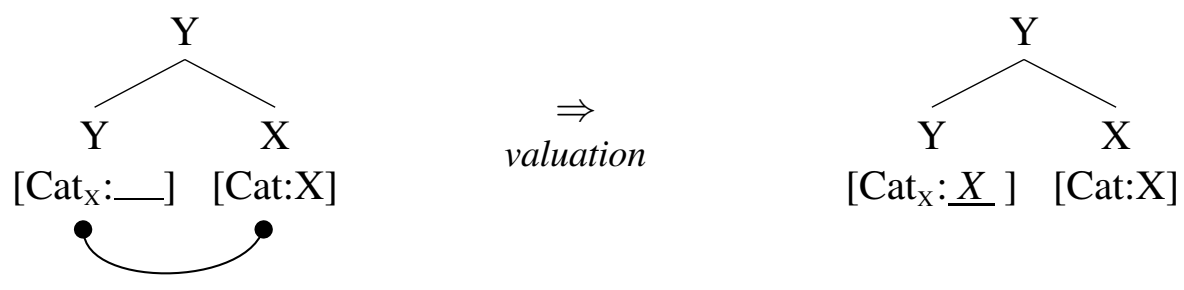
Though the standard assumption is that c-selection triggers Merge, it is at least controversial that c-selection underlies all structure building. Instead, it is often argued that c-selection is only responsible for those instances of structure building that show some level of idiosyncrasy (verbs selecting for particular prepositions, clause types etc.). It seems clear that not every part of clausal structure shows such selectional idiosyncrasy. For instance, functional categories, such as Tense, Aspect, Mood, Negation, tend to come in a fixed order cross-linguistically. These fixed orders of functional categories are referred to as Functional Hierarchies (Cinque, 1999), or Hierarchies of Projections (Adger \& Svenonius, 2011; Ramchand \& Svenonius, 2014). Such hierarchies are treated as universal and not built by selection-triggered Merge - they are pre-defined and fixed. In the light of the dichotomy of syntactic relations - idiosyncratic and universal, Adger (2010) distinguishes two types of structure building, shown in (12).

Two types of structure building (Adger, 2010)

a. Sel-Merge: c-selection-triggered Merge (subcategorization)

b. HoP-Merge: structure building determined by a fixed order of functional projections

Sel-Merge builds structures on the basis of c-selectional features. This type of structure building is what we know as subcategorization and it encodes idiosyncratic relations between the selector and the selectee. The other type of structure building, HoP-Merge, is responsible for creating functional hierarchies, which are universal and not sensitive to c-selectional properties of heads.

Another standard assumption is that c-selection must obtain under sisterhood. The sisterhood configuration of c-selection falls out from a theory where all structure building (i.e. every instance of Merge) is triggered by c-selection. However, this assumption does not take into consideration the possibility of other types of structure building, such as HoP-Merge, which forms functional hierarchies and, crucially, does not rely on c-selection. Adopting this latter view, as I do here, the question arises of what happens when the sisterhood relation is formed by HoPMerge, while at the same time one of the heads has a c-selectional feature. This is a situation where a c-selecting head is also a head belonging to a functional hierarchy. Following Adger (2010) and Adger \& Svenonius (2011), I assume that functional hierarchies are built first and so there can be no projection in the head-complement sequence that does not belong to the hierarchy. This means that c-selection will not trigger merge of a complement if the selecting head is part of a functional hierarchy, as in (13).

\section{A functional hierarchy with a c-selecting head $\mathrm{Y}$}
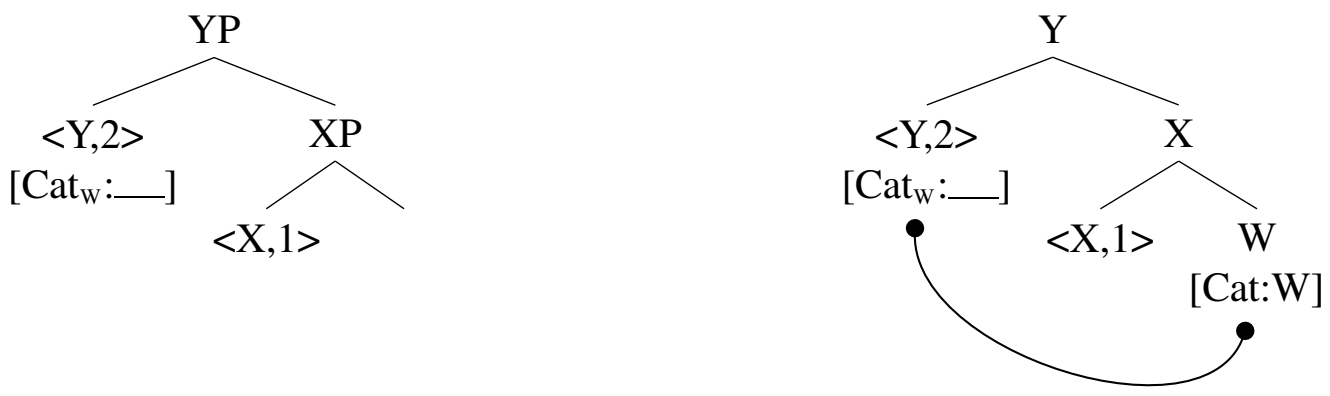

The head $\mathrm{Y}$ in (13) is part of the hierarchy of projections $\langle\mathrm{X}, \mathrm{Y}\rangle$. In addition, $\mathrm{Y}$ c-selects for the category $\mathrm{W}$, but cannot merge $\mathrm{W}$ as its complement since the complement of $\mathrm{Y}$ must be $\mathrm{X}$, 
given the hierarchy. We, then, end up with an HoP-built structure with one unsatisfied selectional feature. An important consequence of formalizing c-selection as Agree is that c-selectional requirements of heads need not be satisfied under sisterhood. Instead, c-selection must obey all and only the locality restriction that the operation Agree is subject to. These involve clause-bound (or phase-bound) locality, but not sisterhood locality. Therefore, the category probe on Y may search deeper into its c-command domain for a matching goal (13)-b.

The view of c-selection as Agree is adopted also by Cowper (2010) in her account of auxiliary distribution in English. The necessity to loosen the strict sisterhood requirement for c-selection has been also pointed out by Svenonius (1994) and Merchant (2016), who show that c-selection exhibits non-local interactions; in particular, the selecting head can be separated from the selected category by other material. Thus, the idea adopted here that selectional features may be satisfied at a distance is independently motivated.

Finally, I assume another possibility of [Cat]-valuation, namely merging a goal as a specifier. This mode of valuation is available only if the probe does not find a matching goal in its ccommand domain - the basic logic behind Cyclic Agree (Béjar \& Rezac, 2009). In other words, unsuccessful [Cat]-probing triggers Merge of a specifier as a last resort mode of valuation (14).

b. Failed downward search

b. Merge of a matching goal
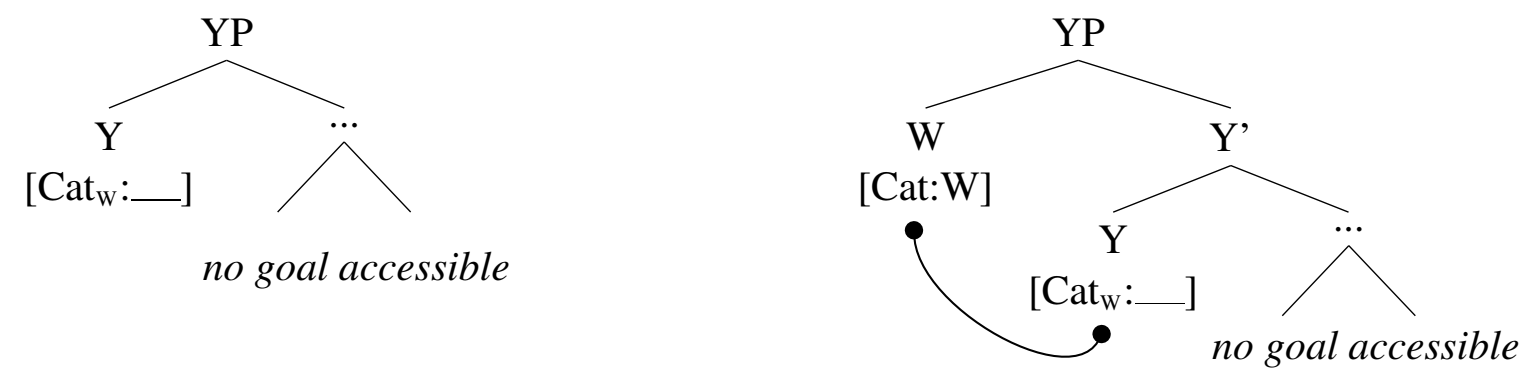

Given that head-complement sequences in a functional hierarchy are built by HoP-Merge, SelMerge (c-selection-triggered Merge) can only produce specifiers (Adger, 2010). This is exactly the consequence of the Cyclic Agree analysis of c-selection: the category goal W is Merged immediately after the unsuccessful downward probing - as a specifier of the selecting head.

3.2. Auxiliary Verb SElection. Auxiliary verbs and main verbs share one obvious property: their category. I propose, following much previous work, that verbs are c-selected by inflectional heads. As discussed in the previous section, I formalize c-selection as Cyclic Agree, whereby probing always involves a search for a matching [Cat]-goal in the c-command domain of the probe, and only in case no goal is found, a matching goal is merged as a specifier of the probing head. In the case of verb selection, the two available modes of valuation of a category probe result in synthesis and periphrasis, respectively. The proposed analysis of auxiliary insertion is, then, based on verb selection (15).

Proposal: The relationship between inflection and (auxiliary) verbs is that of c-selection

a. Main verb: Category agreement by downward probing

b. Auxiliary verb: Category agreement by Merge

The difference between main verbs and auxiliary verbs is that a main verb controls category agreement on a c-commanding functional head under downward probing - the default situation (16)-a, 
while an auxiliary is a reflex of category agreement satisfied by Merge, which takes place only if downward search fails (16)-b. The c-selecting functional head projects and can be targeted by HoP-Merge in further building of the functional hierarchy. ${ }^{4}$

b. Agree (main verb)

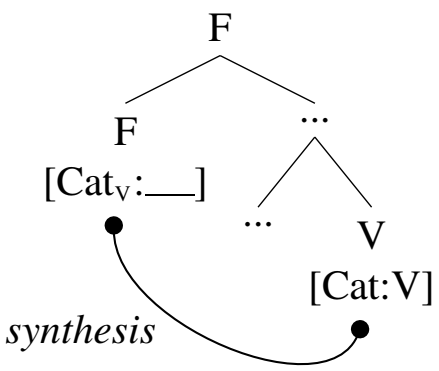

b. Merge and Agree (auxiliary verb)

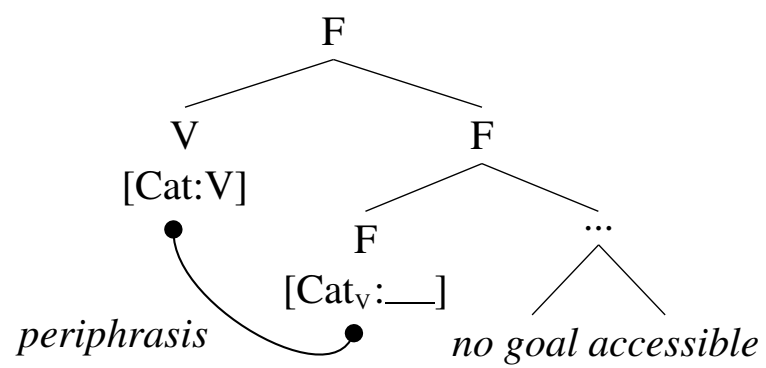

Assuming cyclicity of syntactic operations, downward [Cat]-probing will always apply first, and if successful, will bleed Merge of the selected category. For this reason, as long as a V is accessible for agreement, a new V (the auxiliary) will not be merged, deriving the last-resort profile of periphrasis. ${ }^{5}$

In this section, I developed the basic mechanism of auxiliary insertion as a last-resort merge of a verb triggered by c-selection. The proposed analysis has two crucial properties: i) it can account for the puzzling overflow pattern of auxiliary use, and ii) the auxiliary requirement is understood in terms of the relationship between inflection and verbs in general.

One important question remains: under what circumstances is the main verb inaccessible for the [Cat]-probe on a functional head? In principle, there may be different reasons, likely subject to crosslinguistic variation. In the next section, I propose an analysis of periphrastic tenses in Ndebele in which the inaccessibility of the main verb is due to markedness of tense features and the way Agree-Chains are formed.

4. Deriving auxiliary distribution in Ndebele. In this section, I develop an analysis of perfect and prospective tenses, building on the observation that the pattern of auxiliary use in those tenses has the profile of a last resort mechanism.

4.1. Perfect and Prospective as Tense. Recall that Ndebele synthetic tenses, illustrated in (17-a)-(17-b), contain one tense marker (in a box $)^{6}$. Compound tenses, on the other hand, contain

${ }^{4}$ A further question concerns how the auxiliary combines with the tense morpheme. I assume, following Matushansky (2006), that verbs in a specifier position of inflectional heads can undergo m-merger with that head, resulting in a concatenated or fused form, depending on language.

5 There is an interesting connection between this analysis of auxiliary insertion and the theory of head movement proposed by Matushansky (2006). Note that, after being selected, the auxiliary is a head in a specifier positionthe result of the first step of head movement, in Matushansky's theory. The difference between head movement of a verb and auxiliary insertion reduces to whether the verb is internally merged (movement) or externally merged (auxiliary insertion) in the relevant functional head. The second step of head movement, $m$-merger, can presumably apply in both cases, i.e. whether the configuration required for $m$-merger was obtained by internal or external Merge. This parallel between auxiliary insertion and verb movement is potentially significant - it predicts that auxiliary insertion in a head and verb-movement to that same head are mutually exclusive. This issue is not explored further in this paper.

${ }^{6}$ All examples of past tense in (17) involve Recent Past. This is only for expository reasons - the generalizations and the proposed analysis hold for the Distant Past, as well. 
two: one on the auxiliary and one on the participle (17-c)-(17-e). The future and past tense inflections can combine in either order, future over past and past over future, giving rise to the Future Perfect and the Past Prospective tenses, respectively. When both the auxiliary and the participle bear past tense marking, the resulting tense is Past Perfect (17-e). Note that participles are marked with the same tense affixes as those we find in simple tenses: the perfect participle is formally identical with Simple Past, and the prospective participle is identical with Simple Future.
a. U- $\varnothing-$ dl -ile
Simple Past/Present Perfect (synthetic)
2s- PST- eat -FS.PST
'You ate./You have eaten'.
b. U- Za- dl -a
2s- FUT- eat -FS
'You will eat'.
c. U- za- be u- $\varnothing-$ dl -ile
2s- FUT- aux 2s- PST- eat -FS.PST
'You will have eaten'.
d. U- $\varnothing-$ be u- $\quad$ za- dl -a
2s- PST- aux 2s- FUT- eat -FS
'You were going to eat'.
e. U- $\varnothing$ - be u- $\varnothing$ - dl -ile
2s- PST- aux 2s- PST- eat-FS.PST
'You had eaten'.
Simple Future (synthetic)
Future Perfect (periphrastic)
Past Prospective (periphrastic)
Past Perfect (periphrastic)

I propose that the structure of perfect and prospective tenses contains two T heads. Simple tenses, on the other hand, are structured with one T. Each T bears one of three possible tense features: $\{$ PST, FUT, $\varnothing\}$. I assume that present tense in Ndebele is syntactically unmarked. The notion of syntactic markedness adopted here relies on the presence vs. absence of a feature. For a feature to be syntactically marked simply means to be present. The lack of a present tense feature renders the present tense in Ndebele syntactically unmarked. The generalization about verbal periphrasis in Ndebele is the following: an auxiliary verb is used when the structure contains two tense features, but not when it contains one or none. Thus, the tenses in (18) are all synthetic. Among the perfect and prospective tenses, one other hand, only present perfect is synthetic: the higher T lacks a tense feature, and so there is only one tense feature in this tense - PST on the lower T (19).

Simple tenses:

\begin{tabular}{ll} 
& $\mathrm{T}$ \\
\hline Present Simple & $\varnothing$ \\
Past Simple & PST \\
Future Simple & FUT
\end{tabular}

(19) Perfect/prospective tenses:

\begin{tabular}{lll} 
& $\mathrm{T}_{\mathrm{H}}>$ & $\mathrm{T}_{\mathrm{L}}$ \\
\hline Present Perfect & $\varnothing$ & PST \\
Past Perfect & PST & PST \\
Past Prospective & PST & FUT \\
Future Perfect & FUT & PST
\end{tabular}

The idea that perfect aspect shares some properties with tense is not new and is reflected in proposals which treat the perfect aspect as an instance of tense: T(Anterior) (Cinque, 1999), nonfinite $\mathrm{T}_{\mathrm{PAST}}$ (Hoffman, 1966; McCawley, 1971, 1988; Klein, 1992, 1994; Julien, 2001; Fehri, 2004; Arregi \& Klecha, 2015). According to those analyses, perfect tenses are structured with two tense projections, where the lower (non-finite) one has the value [PAST]. As we saw, the lower T 
in Ndebele is not limited to perfect aspect (i.e. the value [PAST]). Other tense markers, such as the future prefix $z a$ - may appear on the participle, giving rise to what we call prospective aspect. For reasons of exposition, I call the two $\mathrm{T}$ heads $\mathrm{T}_{\mathrm{H}(\mathrm{igh})}$ and $\mathrm{T}_{\mathrm{L}(\mathrm{low})}$, where $\mathrm{T}_{\mathrm{H}}$ corresponds to what we typically understand as tense, while $T_{L}$ is cognate with the projection associate with perfect aspect (or $\mathrm{T}_{\text {Anterior }}$, in Cinque's terms) and prospective aspect (embedded future). Semantic arguments have also been made that perfect tenses involve two temporal relations (Comrie, 1985; von Stechow, 1995; Giorgi \& Pianesi, 1997; Sigurðsson, 2015). In this view, tense and certain types of aspect are inherently homogeneous categories (denoting the notions of temporal anteriority, posteriority etc.), and any differences between them, semantic or morphological, reduce to contextual syntactic factors. The syntactic and semantic connection between tense and perfect/prospective aspect is then supported by Ndebele morphology.

With these assumptions about perfect and prospective tenses, we can move on to developing the details of default periphrasis in Ndebele.

4.2. Agree-Chains AND AUXILIARY SELECTION. Following previous work on periphrasis, I treat the auxiliary as a default lexical item of the verbal category, that is, the simplest bearer of the category feature [Cat: V]. As proposed in section 3, the default verb is selected as a specifier of an inflectional head under two conditions: (i) the head c-selects for a verb and (ii) there is no accessible verb in the c-command domain of the probing head (the Cyclic Agree implementation).

Both $\mathrm{T}_{\mathrm{H}}$ and $\mathrm{T}_{\mathrm{L}} \mathrm{c}$-select for a verb (each has a Cat-probe relativized for $\mathrm{V}$ ), and may have a valued [Tns]-feature (it does in all tenses except in the present tense). The main verb, on the other hand, has a valued [Cat:V] and an unvalued [Tns]-feature. Further, I assume that [Tns] and [Cat] undergo agreement together, as a feature bundle. As an illustration, consider simple tenses, which contain one T:
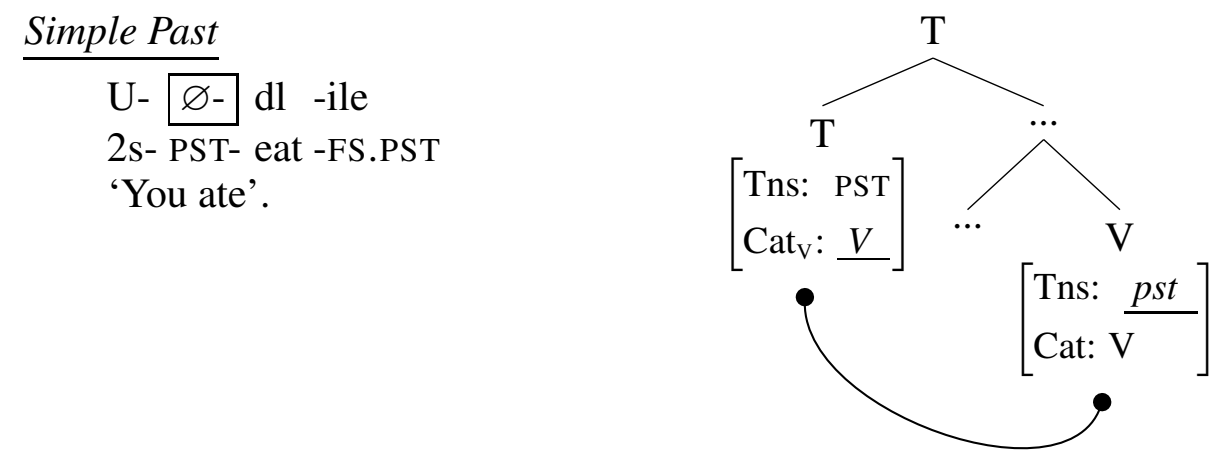

In (20), the category probe on T probes down to find a matching goal: the category feature on the verb. The category probe is valued as [V], establishing an agree relation, or an Agree Chain, between $\mathrm{T}$ and $\mathrm{V}$. As a consequence of [Tns] and [Cat] undergoing agreement as a bundle, the unvalued Tns-feature on the verb receives the value of the selecting inflectional head. I assume that the copied [Tns] feature is realized on the verb as the final suffix (e.g. -ile in (20)). Since simple tenses contain only one T, c-selection involves only one Agree relation. The selectional requirement of $\mathrm{T}$ is satisfied by the main verb and an auxiliary will not be merged in simple tenses.

I adopt the view of agreement as feature-sharing (Brody, 1997; Frampton \& Gutmann, 2000; Pesetsky \& Torrego, 2007) (21). This assumption becomes relevant in the derivation of perfect and prospective tenses, which contain two T heads. 
Agreement as feature sharing (adapted from Pesetsky \& Torrego (2007)):

Formation of an Agree-chain $n$ in which any unvalued instance of a feature is interpreted with the value of a valued instance:

$\mathrm{F}{ }_{[1]} \ldots \mathrm{Fval}_{[2]} \Rightarrow \mathrm{F}{ }_{[2]} \ldots \mathrm{Fval}_{[2]}$

Once two heads establish an Agree relation, they become part of the same Agree Chain or, in Pesetsky \& Torrego's terms, one feature occurrence with multiple instances. As shown in (21), two occurrences of the feature $\mathrm{F}\left(\mathrm{F}_{[1]}\right.$ and $\left.\mathrm{F}_{[2]}\right)$ become one $\left(\mathrm{F}_{[2]}\right)$ under Agree. In this view, valuation is replaced by the notion of feature sharing: $\mathrm{F}_{[1]}$ and $\mathrm{F}_{[2]}$ become one feature under Agree and so they share one value.

Recall that perfect and prospective tenses contain two $\mathrm{T}$ heads. The lower $\mathrm{T}$ is merged first and probes for $[\mathrm{Cat}: \mathrm{V}]$ in its c-command domain (22). The Agree chain $\left[\mathrm{T}_{\mathrm{L}} \ldots \mathrm{V}\right]$ is thus established and it contains a valued instance of [Tns]. Once the higher $\mathrm{T}$ is merged, its unvalued category feature probes down to find a matching goal. However, the verb is already in an Agree chain that contains a value for [Tns]. If $\mathrm{T}_{\mathrm{H}}$ established an Agree-relation with $\mathrm{V}$, the resulting chain would contain two valued [Tns]-features. I propose that chains with more than one value of the same feature are ill-formed since it cannot be determined which value will be shared across its instances. Such offending chains are ruled out by the Condition on Feature Sharing in (24).

Future Perfect (two Tns-features)
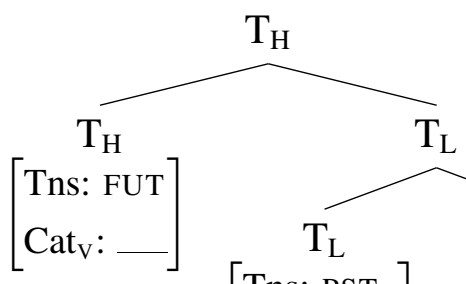

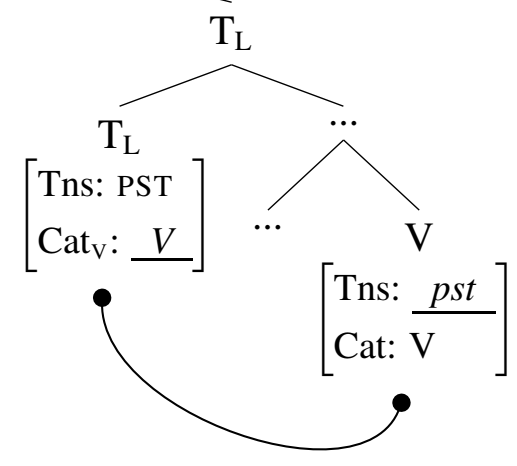

Auxiliary selection:
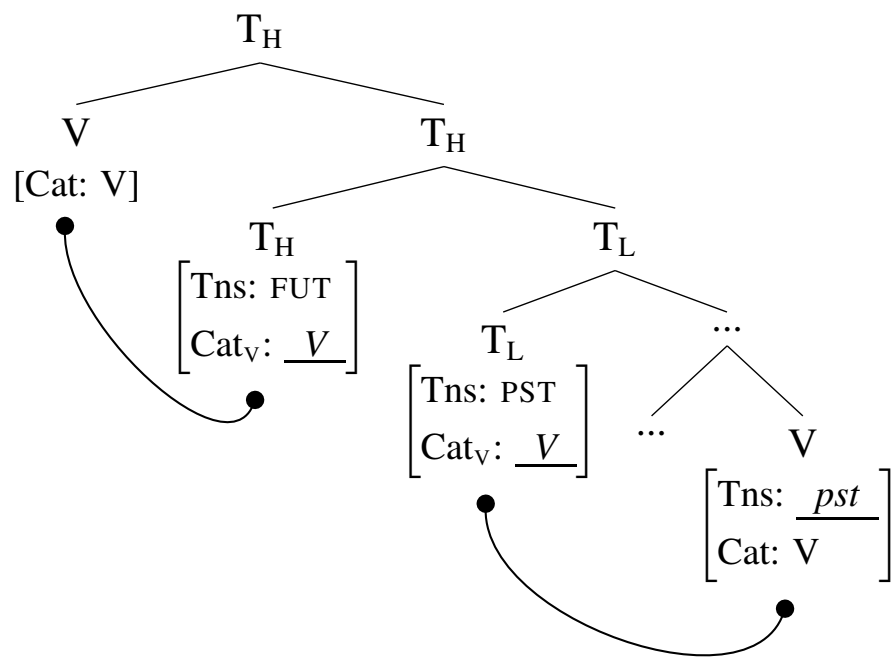

\section{Condition on Feature Sharing}

An Agree-chain $n$ may contain at most one valued instance of a feature.

$* \mathrm{~F} \_[\mathrm{n}] \ldots \mathrm{Fval}_{1[\mathrm{n}]} \ldots \mathrm{Fval}_{2[\mathrm{n}]}$

The Condition on Feature Sharing prevents the higher $T$ from joining the Agree chain $\left[T_{L} \ldots V\right]$ in (22). This is a configuration in which the main verb becomes inaccessible for the [Cat]-probe on a functional head. As a result of Cyclic Agree, the matching category goal is merged as a specifier of the selecting head, $\mathrm{T}_{\mathrm{H}}$, and gives rise to a periphrastic expression (23). Auxiliary selection is derived in the same way in the two other tenses that contain two tense features: Past Perfect and Past Prospective. 
Recall from (17) that, unlike other perfect tenses, the Present Perfect is synthetic. The analysis proposed in this section derives the synthetic form of the Present Perfect: since present tense is syntactically unmarked (has no tense feature), $T_{H}$ does not contribute an extra [Tns] value to the Agree chain. It may, therefore, satisfy its selectional feature by probing the main verb (25).

$$
\begin{aligned}
& \frac{\text { Present Perfect }}{\text { U- } \varnothing-} \text { dl -ile } \\
& \text { 2sg- PST- eat -FS.PST } \\
& \text { 'You have eaten'. }
\end{aligned}
$$

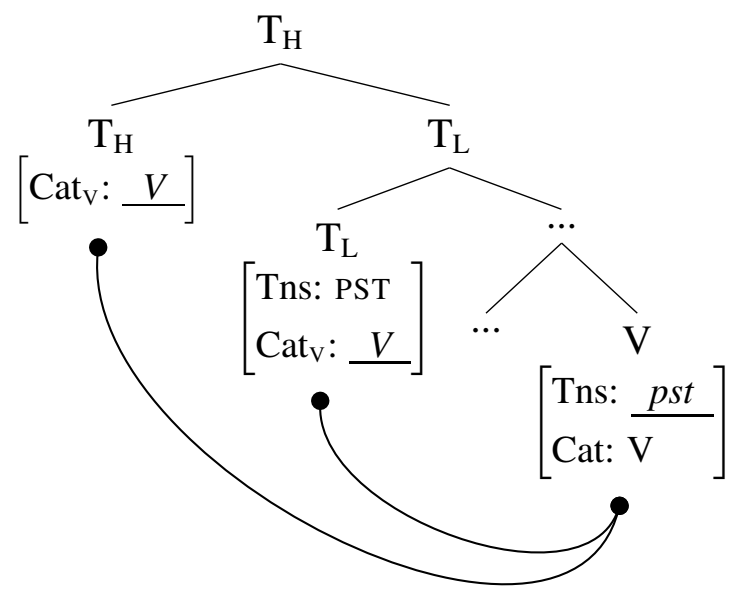

The expression of the Present Perfect is morphologically identical to that of the Simple Past. They are however structurally different - compare (25) and (20). The analysis offered here derives their surface identity. First, T heads with the feature [PST] have a zero exponent and so the number of $\mathrm{T}$ heads cannot be determined by overt morphology. Second, the analysis derives the fact that both are synthetic - there is only one valued [Tns]-feature in each. And third, the final suffix, which covaries with tense, is predicted to have the same form in both tenses - it is valued as [pst] by $\mathrm{T}_{\mathrm{L}}$ in the Present Perfect, or by the sole $\mathrm{T}$ of the Simple Past. ${ }^{7}$

5. Meeting the objectives. Under the present analysis, auxiliary syntax is derived by verb selection in a configuration where the main verb is inaccessible for a category probe on a functional head. The analysis proposed here achieves the same goal as that proposed by Cowper (2010), namely, to provide an implementation of the general intuition that periphrasis is the result of a dependence of an inflection on the verb. In both Cowper's analysis and the analysis proposed here, the dependence is c-selection - inflectional heads must find a verbal element to establish an Agree relation with. In spite of this similarity, Cowper's proposal differs from mine in two important aspects: i) the implementation of last resort and ii) the mechanics of auxiliary insertion.

Cowper implements c-selection as an agreement relation. All inflectional heads have an uninterpretable V-feature which must be checked against a matching interpretable feature [iV] on a verb. In addition, certain inflectional heads share their Infl-features with the verb. Once the verb gets an Infl-feature, it can no longer check [uV]-features on higher inflectional heads (by the Activity Condition). An unchecked uV-feature triggers auxiliary insertion. For instance, the perfect aspect in English renders the verb inactive for further $\mathrm{uV}$-checking, deriving the fact that all perfect tenses in English are periphrastic. In Ndebele, however, not all perfect tenses require an auxiliary - the Present Perfect is synthetic. Such cases are not captured by Cowper's analysis since the perfect would make the verb inactive also in the Present Perfect, predicting auxiliary

${ }^{7}$ A question arises concerning the valuation of [Tns] on the verb in Simple Present, which lacks a [Tns]-feature. I assume that an unvalued [Tns]-feature on $\mathrm{V}$ is spelled out as the default final suffix - $a$. 
insertion in T. Thus, the overflow pattern in Ndebele perfect tenses cannot be straightforwardly derived by Cowper's [uV]-checking system.

As far as the mechanics of auxiliary insertion, an unchecked $\mathrm{uV}$ feature on a head triggers insertion of a verb in that head. This stipulation has the same result as auxiliary selection by Merge, proposed here. The advantage of the present account is that it captures the process of auxiliary insertion in terms syntactic operations independently argued for in the literature.

A more detailed analysis of default periphrasis is offered by Bjorkman (2011), and it specifically targets the understudied overflow pattern of auxiliary use. According to Bjorkman, the overflow distribution eliminates the analytical possibility of projecting an auxiliary in the syntax and of auxiliary verbs being c-selected. Therefore, in Bjorkman's system, Aux-insertion is a response to morphological, rather than syntactic, ill-formedness. Auxiliaries are absent in the narrow syntax altogether and they are inserted in certain inflectional heads at Spellout. The question is, then, how is it determined which inflectional head will be targeted by auxiliary insertion? To answer this question, Bjorkman defines the notion of stranded inflection, which arises in the following way. First, periphrastic forms, such as the Future Perfect, are syntactically well-formed (26)-a. Similarly to my analysis, the verb has an unvalued inflectional feature and all inflectional heads have a valued inflectional feature (e.g. [Fut] or [Perf]). Additionally, all inflectional heads (except the highest one) bear Infl-probes just like the verb. Bjorkman assumes Upward Agree, whereby the values of Infl in (26)-a are transferred one head down: [Infl: Perf] is transferred to the verb, while [Infl: Fut] is transferred to Perf ${ }^{0}$, as shown in (26)-b.

a. Syntax

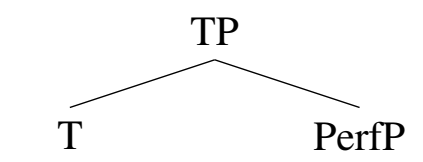

iInfl:Fut

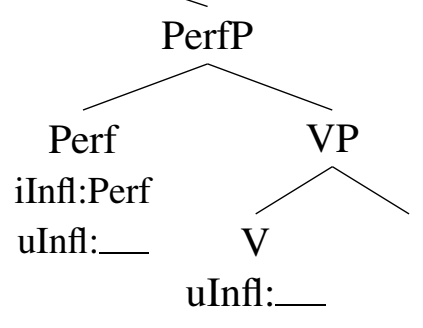

b. Morphology

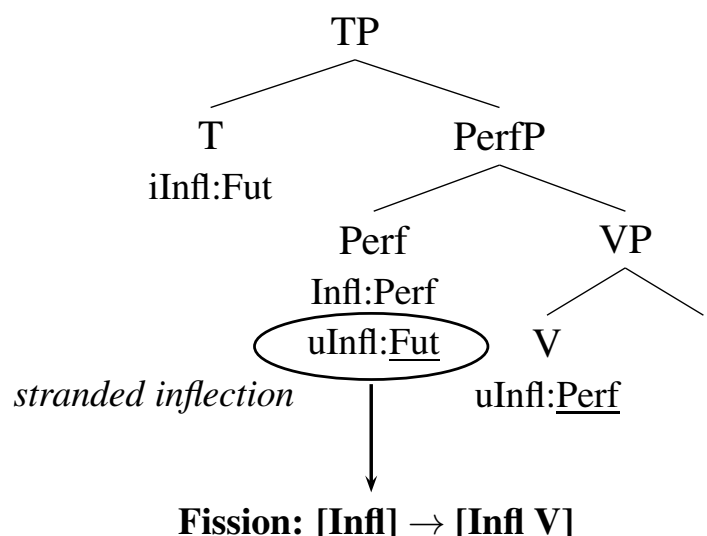

Fission: [Infl] $\rightarrow$ [Infl V]

While this configuration is syntactically well-formed, it poses a violation in the morphology. Following the assumption that inflection requires a verb, Bjorkman proposes that any inflectional head which does not form a complex head with a verb is an offending head called stranded inflection. As we see from (26)-b, the Perf head does not form a complex head with a verb, and therefore qualifies as a stranded inflection. The violation in (26)-b is morphological and it is morphologically repaired. The repair proposed by Bjorkman involves the morphological rule of fission which, in this case, splits the stranded inflection node into itself and a verb.

There are two major problems with this analysis, both of which are avoided under the account advocated in this paper. The first one has to do with the concept of stranded inflection. As intuitive as it seems in the discussion of default periphrasis, the concept of stranded inflection has had no appearance in syntactic theory in any principled way and outside of the literature on pe- 
riphrasis. In fact, the syntactic computation gives rise to a large number of structures that would meet the definition of stranded inflection. It is unclear, for instance, why interpretable Infl features do not count as stranded, e.g. the [iInfl:Fut] in (26). The analysis proposed here derives the same overflow effects without the need to define stranded inflection as a theoretical object that the grammar makes reference to. The second, and perhaps a more serious, issue has to do with the repair mechanism itself. Recall that one of the major objectives of this paper was to understand why the failure of an inflection to combine with a verb results in insertion of another verb. Bjorkman gives two unrelated answers to this question: Inflection combines with a verb in the syntax by Agree, and in the morphology by a fission rule. This analytical choice gives up the objective set up here entirely.

6. Conclusion. This paper addressed two questions about default periphrasis: i) What property of the grammar requires inflection to combine with a verb? and ii) What property of the grammar is responsible for the appearance of auxiliaries? The goal was to find a single answer to both questions, that is, to identify the formal connection between inflections and verbs and explain the mechanics and rationale of auxiliary insertion in terms of this formal relation. I proposed an analysis of auxiliary insertion as a derivational phenomenon. Syntactic computation determines whether a particular inflectional context will give rise to synthesis or periphrasis. The main advantage of the proposed account is the fact that it naturally derives overflow patterns of auxiliary use, avoiding a stipulation about how and why auxiliaries are inserted: just like lexical verbs, auxiliary verbs are c-selected and merged during syntactic structure building. Formalizing c-selection as the Agree operation (a proposal made independently in the literature) allows inflectional heads to select for verbs under a less local configuration than sisterhood. Combining this approach to c-selection with the Cyclic Agree theory of agreement gives rise to a system where auxiliary verbs are selected and merged as specifiers if and only if the selecting inflectional head cannot agree with the main verb, deriving the last-resort property of default periphrasis. Moreover, the cyclic selection system developed here allows us to avoid making reference to the concept of stranded inflection. Under the present analysis, stranded inflection may still be used as a descriptive term, but it is not a primitive theoretical object.

\section{References}

Adger, David. 2010. A minimalist theory of feature structure. In Features: Perspectives on a key notion in linguistics, Oxford University Press.

Adger, David \& Peter Svenonius. 2011. Features in minimalist syntax. The Oxford handbook of linguistic minimalism 27-51.

Arregi, Karlos \& Peter Klecha. 2015. The morphosemantics of past tense. In Proceedings of the 45th conference of the North East Linguistics Society, .

Béjar, Susana \& Milan Rezac. 2009. Cyclic Agree. Linguistic Inquiry 40(1). 35-73.

Bjorkman, Bronwyn Alma Moore. 2011. BE-ing default: The morphosyntax of auxiliaries: Massachusetts Institute of Technology dissertation.

Brody, Michael. 1997. Perfect Chains. In Elements of grammar, 139-167. Springer.

Chomsky, Noam. 1993. A minimalist program for linguistic theory. In Kenneth Hale \& Samuel Jay Keyser (eds.), The View from Building 20: Essays in Linguistics in Honor of Sylvain Bromberger, 1-52. MIT Press. 
Cinque, Guglielmo. 1999. Oxford Studies in Comparative Syntax chap. Adverbs and functional heads: A cross-linguistic perspective. Oxford University Press.

Comrie, Bernard. 1985. Tense, vol. 17. Cambridge University Press.

Cowper, Elizabeth. 2010. Where auxiliary verbs come from. In Proceedings of the 2010 annual conference of the Canadian Linguistic Association, .

Déchaine, Rose-Marie. 1995. One be. Linguistics in the Netherlands 12(1). 73-88.

Fehri, Abdelkader Fassi. 2004. Temporal/aspectual interaction and variation across Arabic Heights. In Jacqueline Guéron \& Jacqueline Lecarme (eds.), The Syntax of Time, MIT Press.

Frampton, John \& Sam Gutmann. 2000. Agreement is feature sharing. Unpublished manuscript. Northeastern University. http://babel. ucsc . edu/ hank/mrg.readings/agrisfs .pdf .

Giorgi, Alessandra \& Fabio Pianesi. 1997. Tense and Aspect: From Semantics to Morphosyntax. Oxford University Press.

Hoffman, Ronald. 1966. Past tense replacement and the modal system. In Anthony Oettinger (ed.), Mathematical linguistics and automatic translation, Cambridge, MA: Harvard University, Harvard Computation Laboratory.

Iatridou, Sabine, Elena Anagnostopoulou \& Roumyana Izvorski. 2003. Observations about the form and meaning of the Perfect. In Artemis Alexiadou, Monika Rathert \& Arnim von Stechov (eds.), Perfect Explorations, 153-204. Mouton de Gruyter.

Julien, Marit. 2001. The syntax of complex tenses. The Linguistic Review 18. 125-167.

Klein, Wolfgang. 1992. The present perfect puzzle. Language 525-552.

Klein, Wolfgang. 1994. Time in language. London: Routledge.

Matushansky, Ora. 2006. Head movement in linguistic theory. Linguistic Inquiry 37(1). 69-109.

McCawley, James. 1971. Tense and time reference in english. In Charles Fillmore \& D. Terence Langendoen (eds.), Studies in linguistic semantics, 96-113. New York: Holt, Rinehart and Winston.

McCawley, James. 1988. The syntactic phenomena of english. Chicago: University of Chicago Press.

Merchant, Jason. 2016. Roots don't select: A novel argument from category-dependent 1selection. Talk given at: The 42nd meeting of the Berkeley Linguistics Society, University of California, Berkeley, 5 February 2016.

Pancheva, Roumyana. 2003. The aspectual makeup of perfect participles and the interpretations of the perfect. In Artemis Alexiadou, Monika Rathert \& Arnim von Stechov (eds.), Perfect Explorations, 277-306. Mouton de Gruyter.

Pesetsky, David \& Esther Torrego. 2007. The syntax of valuation and the interpretability of features. Phrasal and clausal architecture: Syntactic derivation and interpretation 262-294.

Ramchand, Gillian \& Peter Svenonius. 2014. Deriving the functional hierarchy. Language Sciences 46. 152-174.

Rothstein, S. 2004. Predicates and their subjects. Springer.

Rothstein, Susan. 1999. Fine-grained structure in the eventuality domain: The semantics of predicative adjective phrases and be. Natural Language Semantics 7. 347-420.

Schütze, Carson. 2003. When is a verb not a verb? Nordlyd 31. 400-415.

Sigurðsson, Halldór Ármann. 2015. The split T analysis. Finiteness Matters .

von Stechow, Arnim. 1995. On the proper treatment of tense. In Proceedings of Semantics and

Linguistic Theory, vol. 5, 362-386.

Svenonius, Peter. 1994. C-selection as feature checking. Studia Linguistica 48(2). 133-155. 